\title{
Information-Geometric Lenses for Multiple Foci+Contexts Interfaces
}

\author{
Richard Nock* \\ CEREGMIA - U. Antilles-Guyane, France
}

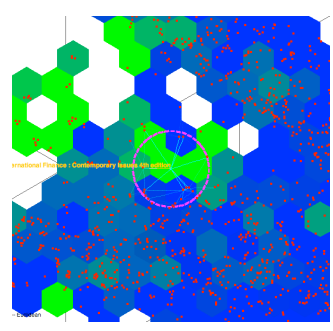

Euclidean

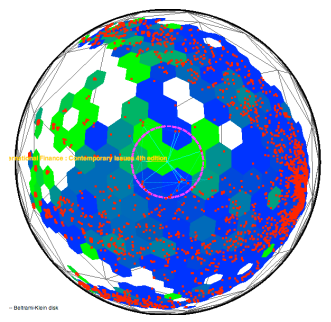

Beltrami-Klein

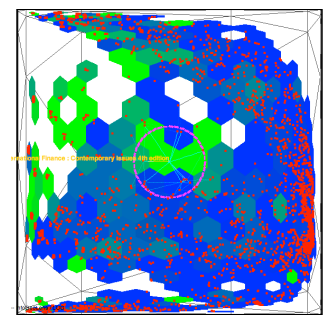

InfoGeoLens(LOG)

\author{
Frank Nielsen $^{\dagger}$ \\ SONY CS Labs, Inc., Japan
}

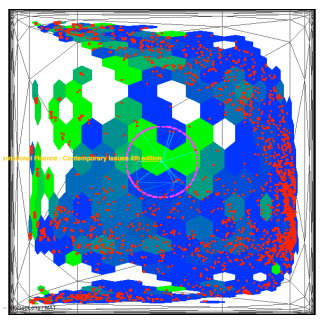

InfoGeoLens(MAT)

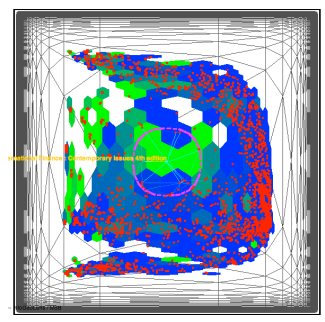

InfoGeoLens(MSB)

Figure 1: Browsing a large-scale digital library requires tailored interfaces: Euclidean does not display full context, Beltrami-Klein (or Poincaré) do not fit rectangular displays (Poincaré is conformal). InfoGeoLenses have several advantages: (i) they fit rectangular displays, (ii) their focus region is approximately Euclidean, (iii) they keep tangible shapes and (iv) they authorize multiple foci+contexts (see text).

\begin{abstract}
We present a new set of 2D/3D modeling and visualization techniques that build upon recent information geometric works, with desirable properties like seamless multiple foci+contexts abilities, several keeping of meaningful topological features and tangible shapes, and a very good Euclidean approximation near the focus, which make them reliable candidates to display (geographic) maps or pictures. We show that a slight modification of a popular fisheye view, namely Sarkar-Brown's, belongs to this set. We report on two experiments on $2 \mathrm{D}$ and $3 \mathrm{D}$ interfaces against contenders from hyperbolic geometry. It is a browsing task involving a real-world virtual library, whose map is a manifold learned from the traces of $60 \mathrm{k}+$ users, and consisting of approximately 10k books. Observations and users' feedback suggest that information geometry makes a sound alternative to hyperbolic geometric approaches, and may help to craft appealing geometric focus+context interfaces tailored to specific displays or domains.
\end{abstract}

CR Categories: H.5.2 [Information Interfaces and Presentation]: User Interfaces: Graphical User Interfaces, Theory and MethodsTheory and Methods

Keywords: Focus+Context, Information Geometry

\section{Introduction}

Today, it is becoming easier and easier to observe and capture the infinitely large with commodity hardwares, yet it is becoming harder in the meantime to find accurate techniques to render data on conventional displays [Pindat et al. 2012]. But fitting the very large scale to conventional displays is not the only challenge. At the other extreme of the resolution scale, visualizing the infinitely small

\footnotetext{
*e-mail:rnock@martinique.univ-ag.fr

†e-mail:nielsen@acm.org
}

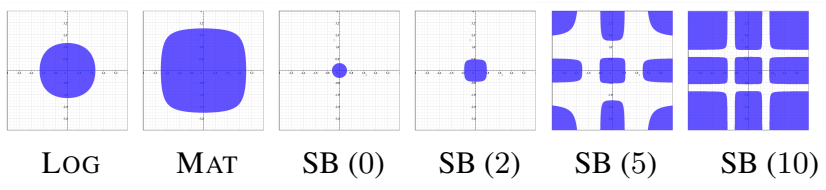

Figure 2: Mapping of an euclidean disk centered around the focus in a focus +context view, back into the euclidean plane. InfoGeoLenses (LOG, MAT) guarantee to keep a connex and convex surface, while neither is guaranteed for the original Sarkar-Brown's fisheye for large values of the magnification factor (SB, magnification factor $d$ is indicated in parenthesis; see text for details).

asks for similar challenges. For example, a recent workflow of techniques from biology and systems biology produces the equivalent of geographical maps in which cities are genes, neighboring genes have more significant interactions, and roads between genes denote meaningful correlations in expression recorded "at run time" [Matsuoka et al. 2009]. Using these techniques, the map obtained from barely visible creatures like Daphnia pulex would be much larger and more complex than the United States' map.

Focus+context techniques provide a convenient way to visualize such highly complex maps or pictures, by providing the user with the display of a detailed region of interest along with its surrounding context [Cockburn et al. 2008]. A prominent example of such techniques are fisheye (lens) views, initially born from the optical properties of media [Furnas 2006; Furnas 1986; Sarkar and Brown 1992], and extensively used so far to represent hierarchies [Lamping et al. 1995]. Most available focus+context interfaces rely on physical analogies or ad hoc mathematical approaches [Pindat et al. 2012].

A fundamental difference between hierarchies and (geographic, picture) maps is that the latter require more care to preserve topological and geometric cues through focus+context view, cues that can be helpful to understand and handle the final, distorted map. A close inspection at various focus+context interfaces reveals that if we filter out those that do not manage simple invariants on geometric primitives, then we barely finish up with "canonical" focus+context representations born from hyperbolic geometry, like Poincaré or Beltrami-Klein disks, that have been inspiring for a very long time in the field [Furnas 2006; Furnas 1986]. Let us 


\begin{tabular}{|c|c|c|}
\hline \hline & $f(x): \mathbb{R} \rightarrow[0,1]$ & $f^{-1}(x):[0,1] \rightarrow \mathbb{R}$ \\
\hline \hline LOG & $\frac{\exp (r x)}{1+\exp (r x)}$ & $\frac{1}{r} \ln \left(\frac{x}{1-x}\right)$ \\
\hline MAT & $\frac{1}{2}\left(1+\frac{r x}{\sqrt{1+(r x)^{2}}}\right)$ & $\frac{1}{r} \frac{2 x-1}{\sqrt{x(1-x)}}$ \\
\hline MSB & $\frac{1}{2}\left(1+\frac{r x}{1+r|x|}\right)$ & $\begin{array}{l}\frac{1}{2 r}\left(\frac{x}{1-x}-1\right) \text { iff } x>\frac{1}{2} \\
\frac{1}{2 r}\left(1-\frac{1-x}{x}\right) \text { otherwise }\end{array}$ \\
\hline
\end{tabular}

Table 1: Three different InfoGeoLenses: name (left), permissible mapping $f$ (center), and its inverse $f^{-1}$ (right); MSB stands for "Modified Sarkar-Brown"; $r>0$ is the distortion factor (see text).

illustrate this with a simple shape problem. Humans readily perceive shapes in complex scenes: how does an euclidean disk in the focus+context interface maps back into the natural, euclidean space ? Figure 2 provides an example for a radius- 4 euclidean disk centered around the focus, mapped back with Sarkar-Brown's fisheye, a popular focus+context approach [Cockburn et al. 2008; Sarkar and Brown 1992], with various values for the distortion factor $r$ (the four rightmost plots in Figure 2). One sees that when $r$ increases, the euclidean shape loses both the connexity and convexity of the disk, which may impair the interpretation of the focus+context view and prevents large zooming. Poincaré or Beltrami-Klein disks (not shown) do not suffer such problems, yet they present other drawbacks, like counterintuitive behaviors (e.g. translations become rotations) [Lamping et al. 1995], or the fact that having circular shapes makes them not the best candidates for rectangular displays ${ }^{1}$. It is the purpose of this note to propose an alternative to hyperbolic geometry for focus+context interfaces, rooted in information geometry: Information Geometric Lenses (InfoGeoLenses). The two leftmost pictures in Figure 2 present the mapping of the disk for two examples of InfoGeoLenses. Both (LOG, MAT) preserve the convexity and connexity of the disk, a property that holds for all InfoGeoLenses we consider.

The following section presents InfoGeoLenses. It is followed by a section showing how to render them, and reporting on a controlled experiment of both 2D and 3D InfoGeoLenses, including comparisons with Poincaré and Beltrami-Klein disks.

\section{Information Geometric Lenses}

At first glance, information is a protean and ubiquitous material, yet it does not come without structure in first place. Pioneering works by S.-i. Amari have progressively shown that information obeys particular non-Euclidean geometries of arbitrary dimension [Amari and Nagaoka 2000], different from Poincaré or BeltramiKlein disks'. A most important property states that one can embed the natural, ambient space, into a dual space with sizable advantages related to modeling, rendering and visualizing information. We list some of these advantages.

a-Closedness of the dual space The space in which we zoom may be closed, e.g. $[0,1]^{n}$ (see Figure 3 ), which is particularly interesting for visualization purposes. For simplicity, we restrict ourselves hereafter to mappings for which the dual space is $[0,1]^{n}$.

b - Focus+context behavior of the connection map In this case, there exists a bijective mapping $f$ between the two, which maps any real coordinate $x_{i}$ into $f\left(x_{i}\right) \in[0,1]$, also called a connection map. $f$ is permissible: its inverse, $f^{-1}$, is the derivative

\footnotetext{
${ }^{1}$ This can be fixed with coordinate-wise hyperbolic transformations [Hovestadt et al. 1995], but at the expense of the lost of other properties.
}

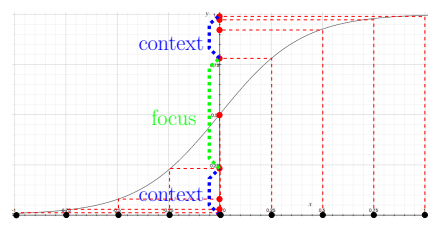

Figure 3: Mapping regularly spaced black dots of the real line onto the logistic interval $[0,1]$ via the permissible $f$ of the InfoGeoLens LOG with distortion factor $r=5$ (see Table 1) produces a set of non-regularly spaced red dots, which displays a focus around the center red dot (in green). The blue regions denote the context.

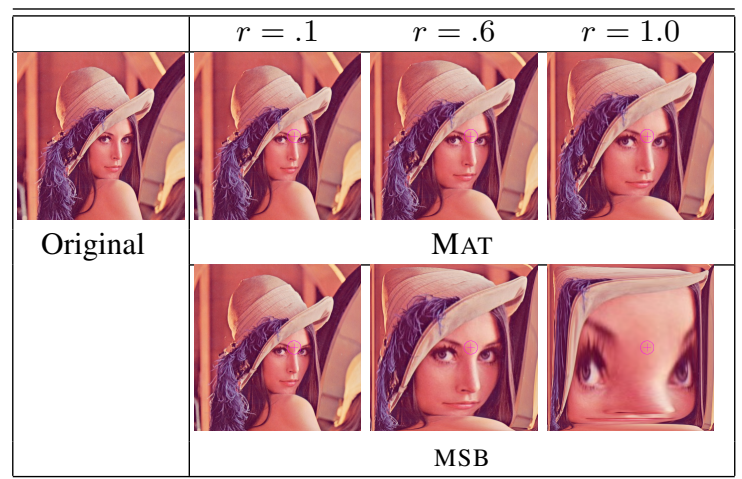

Figure 4: A picture (left-up, 512×512), mapped in InfoGeoLens LOG (up) and MSB (bottom), for different values of $r$. The center of the zoom (i.e. the center of the focus area), indicated by a magenta target sign, is between the eyes.

of a strictly convex function symmetric around $x=1 / 2$ [Nock and Nielsen 2009]. Table 1 presents three examples of permissible mappings $f$. Distortion factor $r$ controls the magnification. The top-most example (LOG) is the popular logistic map. The second (MAT) is Matsushita's map [Nock and Nielsen 2009]. The bottommost example results from a slight modification of Sarkar-Brown's fisheye map [Sarkar and Brown 1992], namely the insertion of the absolute value in the denominator (plus cosmetic scaling details). This modification guarantees that the "Modified Sarkar-Brown" is now an InfoGeoLens, and does not suffer the drawbacks displayed in Figure 2. Moreover, looking closely at the expressions of $f^{-1}$ in Table 1, one may see that the Modified Sarkar-Brown mapping is the first-order approximation of the logistic mapping (once again, modulo cosmetic scaling details). The original Sarkar-Brown fisheye is thus very close to information geometric mappings. All examples of Table 1 display the same focus (image by $f$ of reals near the origin) + context (image of reals "far away" from the origin) behavior, exemplified in Figure 3 for the LoG InfoGeoLens.

c - The region near the focus is approximately Euclidean The vicinity of the focus center has a geometry which is approximately Euclidean: in the vicinity of center $c, f(y)$ is approximately $(y-c)^{2} f^{\prime}(c) / 2$, which is thus Euclidean with conformal factor $f^{\prime}(c) / 2$. Thus, even when the context gets more and more distorted as $r$ increases, the perception of the focus region may remain within tangible, "Euclidean" bounds for the user. This is exemplified on a picture in Figure 4. One can see that at all distortions, the focus region has negligible distortion compared to the original image, and appears as a conventional zoom over the picture's central region. This is also exemplified on a map in Figure 1: the automatic magnification shows that the focus regions in all InfoGeoLenses are similar to the Euclidean display, with the image of the focus circle 


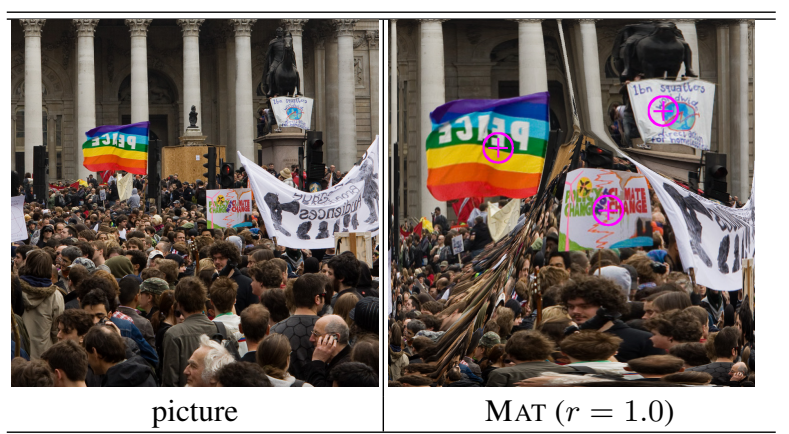

Figure 5: Three focus+context mappings on an image (left, $512 \times 512$ ), where the foci are indicated by magenta target signs. Remark the seamless deformations of the Voronoi cells.
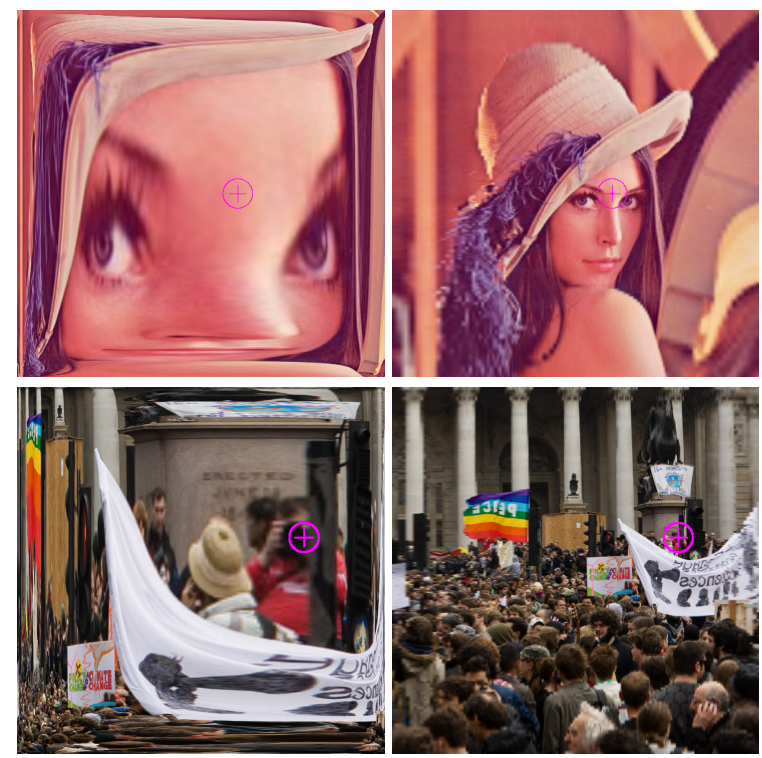

Figure 6: Mapping back zoomed images (left column, MSB, $r=$ 1.0) by $f^{-1}$ (right column). Differences are very slim with the original images (Figures 4 and 5), and located far away from the foci.

(magenta) of near circular shape in LOG and MAT.

d - Meaningful topological features and shapes are preserved Two essential topological properties of the connection map are (i) it is not necessarily conformal - i.e. it does not preserve angles, unlike e.g. Poincaré disk embedding -, but (ii) the connection map does not change Voronoi diagrams [Boissonnat et al. 2010]. Hence, it does not alter the basic topological features of point sets, and keeps tangible shapes as well. Moreover, the information's associated distortion, which is not a metric [Amari and Nagaoka 2000], defines shapes that resemble and match properties of their Euclidean equivalent for the simplest ones, like balls, regular polyhedra, etc. [Boissonnat et al. 2010]. For example, information balls would be convex for all our connection maps $f$.

e - Simple multiple foci+context from foci+zooms only Several works have focused on approaches to zooming that allow for simultaneous zooms on regions of interest in the image [Tobita 2012]. These approaches proceed by mapping the image from a regular grid to a deformed mesh which is supposed to encode the
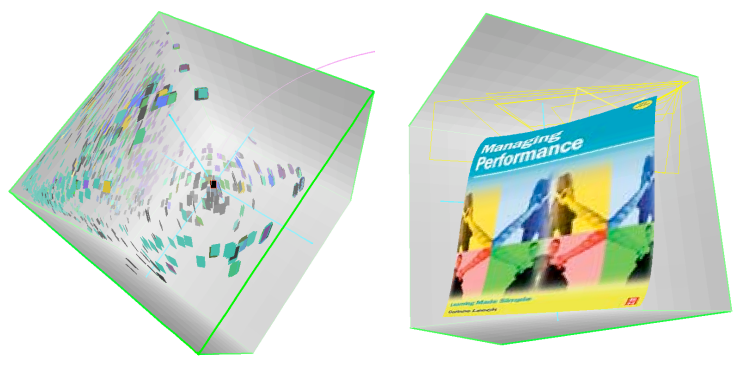

Figure 7: Perspective rendering of InfoGeoLens LOG, on the virtual library. Focus is the cube's center. There are 2 ways to display a book: ( $i$ ) a filled rectangle, colors indicating Dewey subjects (left, all books), or (ii) its cover, rendered in the InfoGeoLens (right).

subregions to zoom in. These approaches have the drawback that zooming becomes a complex and heuristic task, with mesh deformation to design. With InfoGeoLenses, it it very simple to extend the technique by providing the foci only, and the zooming factor. To do this, we independently carry out the focus+context mappings in the voronoi diagram of the foci, as shown in Figure 5.

f - Simple and almost lossless reconstruction of the original image Sometimes, a "good" zoom is defined as a zoom which loses the least possible information from the original image [Tobita 2012]. Let us define a good zoom as one which allows to retrieve an image close to the original, using the least possible information from the zoomed image only. In our case, because the permissible mappings are invertible, knowing the mapping $f$, the foci and the distortion factor $r$ is enough to retrieve an estimate of the original image, in reduced time furthermore, by computing or approximating $f^{-1}$. Figure 6 displays that we indeed retrieve the original almost perfectly for the region near the foci. The map back is also of very good perceptual quality for regions far away from foci.

\section{Rendering and using InfoGeoLenses}

Figures 1 and 7 respectively display 2D and 3D rendering interfaces of InfoGeoLenses. These were the basis of two experiments conducted on a virtual library, used by $60000+$ readers (mainly scholars, students and library patrons) and containing 9980 books dealing with science, travel, business and cooking ${ }^{2}$. The library was learned using manifold learning techniques, that provide the virtual $2 \mathrm{D}$ or $3 \mathrm{D}$ positioning of books in the library, that meet sophisticated placement requirements, and is freed from the physical constraints of their paper twins' positioning [Nock et al. 2013].

\subsection{The 2D interface}

We compared all InfoGeoLenses of Table 1, and two contenders from hyperbolic geometry: Poincaré disk [Lamping et al. 1995] and Beltrami-Klein disk. This latter disk is less used than the former, but it has the major feature that straight lines in the plane get mapped to line segments, instead of arcs in Poincaré disk. The leftmost picture in Figure 1 presents the 2D Euclidean rendering of the virtual library, in a simplified way to ease reading — in the actual interface, covers of books near the focus are displayed. It also presents the five focus+context views. In Figure 1, red points denote books; blue/green hexagons indicate their book's main language; an Euclidean circle in magenta is mapped into each interface: it is centered around a book ("International Finance : Contemporary Issues

\footnotetext{
${ }^{2}$ http: //www.scholarvox.com/?sitelang=en
} 
4th ed.") and indicate its bookshelf neighbors. In the background, the image of a regular triangular tiling is displayed in dark grey. Once started, each view was automatically magnified - thus yielding the value of $r$ in Table $1-$ so that the distance between the two books closest to the center of the square, i.e. the focus, is the same for all interfaces (25 pixels in Figure 1). One of these two books is the book in focus. Remark that LOG provides the largest focus region and MSB the smallest one. The 2D interface was used in a simple experiment involving 9 scholars unfamiliar with focus+context interfaces, and without knowledge of the mathematical foundations behind each one we used. Each scholar was allowed to manipulate (drag-drop, zoom) the interface and was first asked whether each interface was 2D or 3D. Answers were immediate and wrong for both Poincaré (not shown in Figure 1 to keep readable plots) and Beltrami-Klein disks, mistaken for 3D balls. Comparatively, 6 out of 9 answers were wrong for the InfoGeoLens. Users were thus less misled by wrong analogies, and appreciated the intuitive behavior of InfoGeoLenses for simple transformations like translations.

\subsection{The 3D interface}

We carried out an experiment on a full 3D perspective rendering of the InfoGeoLens interfaces, in which book covers were also rendered, as displayed in Figure 7. The objective was to lift some of the 2D observations to 3D, and in particular address whether users' handling of InfoGeoLenses was still as simple as for 2D. The 3D InfoGeoLens is equipped with rotation and magnification that could be performed inside or outside the cube: outside magnification and rotation follow Euclidean rules, while the inside ones follow the InfoGeoLens behavior (see the companion video). For example, the important inside magnification in the right plot of Figure 7 distorts the book's cover - yet, it keeps it highly readable. We carried out a simple 2 within-subject design with the single factor being the Euclidean/LOG interface, on a benchmark of 57 undergraduate students in economics and marketing during a lab session. The interfaces, implemented in Java, were used on browsers to (i) locate a book - a query pinpoints the book, and the student rotates the library to put this book in the foreground - , (ii) the student clicks on the book, which provides an animation of the book moving towards the center of the browser following a shortest path to the center, (iii) repeat the two steps for other queries. Students were asked at the end of the session with which of the two browsers navigation among books was the easiest. Both interfaces were initialized with the same 3D viewpoint. A majority of 49 out of 57 (i.e. more than $73 \%, \alpha=.05$ ) favored LOG, immediately or in the long run. Students were also very interested by the two kinds of rotations. We witnessed during the experiments surprisingly few misinterpretations of the library's topology, a fact that we interpret as coming from the smoothness of inside/outside rotations, also preserving the global topology of data.

\section{Discussion and conclusion}

Hyperbolic geometry has been a fruitful source of inspiration to craft focus+context techniques over the last twenty years [Lamping et al. 1995; Sarkar and Brown 1992]. A significant part of the core theory of information geometry has been developed in the meantime [Amari and Nagaoka 2000]. We do believe that it does not only provide us with neat foundations for the abstract nature of information: it also brings concrete and elegant ideas to display information. An observation strengthens this position: the heavily used Sarkar-Brown's fisheye view is an approximation to such displays.

As a first step in this direction, we have presented in this brief InfoGeoLenses, a general set of focus+context views inspired by information geometry, which brings several interesting guarantees for modeling and rendering (geographic, picture) maps on an interface. Preliminary experiments yield that InfoGeoLenses bring an interesting alternative to contenders inspired by hyperbolic geometry [Lamping et al. 1995]. InfoGeoLenses are also simple to implement, and require in general modest computational resources. They are also versatile: the focus area can have numerous shapes depending on the connection map $f$. InfoGeoLenses make an interesting contender to the Elastic Presentation Framework (EPF) of [Carpendale and Montagnese 2001], as neither of these two sets belongs to the other. The basis of the EPF framework is indeed Euclidean, as it roughly consists in observing a (generally) non-linear lift of a $2 \mathrm{D}$ map in $3 \mathrm{D}$, capturing distances from viewpoint with some fixed metric. The natural distortions associated to information geometry are not metrics, as they are in general not symmetric, nor do they obey the triangular inequality [Amari and Nagaoka 2000]. Blending both frameworks for improved lenses is an interesting problem.

\section{Acknowledgments}

Work done in part while R. Nock was visiting Sony CS Labs, Inc. .

\section{References}

AMARI, S.-I., AND NAGAOKA, H. 2000. Methods of Information Geometry. Oxford University Press.

Boissonnat, J.-D., Nielsen, F., And Nock, R. 2010. Bregman Voronoi Diagrams. DCG 44, 2, 281-307.

Carpendale, S., And Montagnese, C. 2001. A framework for unifying presentation space. In $14^{\text {th }}$ UIST.

Cockburn, A., Karlson, A., And Bederson, B.-B. 2008. A review of overview+detail, zooming, and focus+context interfaces. ACM Computing Surveys 41, 1-31.

FURnAs, G.-W. 1986. Generalized fisheye views. In $4^{\text {th }} \mathrm{CHI}$, 16-20.

FURNAS, G.-W. 2006. A fisheye follow-up: Further reflections on focus+context. In $24^{\text {th }} \mathrm{CHI}, 999-1008$.

Hovestadt, V., Gramberg, O., and Deussen, O. 1995. Hyperbolic user interfaces for computer aided architectural design. In $13^{\text {th }}$ CHI, 304-305.

LAmping, J., RaO, R., And Pirolli, P. 1995. A focus+context technique based on hyperbolic geometry for visualizing large hierarchies. In $13^{\text {th }} \mathrm{CHI}, 401-408$.

Matsuoka, Y., Shoemaker, J.-E., Polouliakh, N., MuRAmoto, Y., Fujil, K., Ghosh, S., Nock, R., Nielsen, F., KAWAOKA, Y., AND KitANO, H. 2009. A systems biology approach to influenza virus infection. In $10^{\text {th }}$ ICSB.

Nock, R., AND NiElSEN, F. 2009. Bregman divergences and surrogates for learning. IEEE T. PAMI 31, 2048-2059.

Nock, R., NiElsen, F., AND BRIYs, E. 2013. Non-linear book manifolds: learning from associations the dynamic geometry of digital libraries. In $13^{\text {th }}$ ACM/IEEE JCDL, 313-322.

Pindat, C., Pietriga, E., Chapuis, O., And Puech, C. 2012. Jellylens: Content-aware adaptive lenses. In $25^{\text {th }}$ UIST.

SARKAR, M., AND BROWN, M. 1992. Graphical fisheye views of graphs. In $10^{\text {th }} \mathrm{CHI}, 83-91$.

ToBita, H. 2012. Contents enforme: Automatic deformation of content for multi-features without information loss. In ACHI'12, 135-140. 